\title{
Anisotropic Phase Field Model of Heteroepitaxial Growth
}

\author{
Dieu Hung HoAng ${ }^{a, *}$, M. BeneŠ ${ }^{a}$, J. StráskÝ ${ }^{b}$
}

${ }^{a}$ Department of Mathematics, Faculty of Nuclear Sciences and Physical Engineering,

Czech Technical University in Prague, Trojanova 13, Prague, Czech Republic

${ }^{b}$ Department of Physics of Materials, Faculty of Mathematics and Physics, Charles University,

Ke Karlovu 5, Prague, Czech Republic

\begin{abstract}
We study the heteroepitaxial growth of thin layers by means of the modified phase-field model with the incorporated anisotropy. The influence of elastic and surface energies on the layer growth is considered. For numerical solution of the model, an explicit numerical scheme based on the finite element method is employed. The obtained computational results with various anisotropy settings demonstrate the anisotropic thin-layer pattern growth.
\end{abstract}

DOI: $10.12693 /$ APhysPolA.128.520

PACS: 81.10.Aj, 05.70.Ln, 81.40.Jj, 81.30.Fb

\section{Introduction}

Heteroepitaxy is an important technology for the contemporary electronic and photo-electric devices. Understanding the physics underlying heteroepitaxial growth is crucial for development of new technologies. It is well known that epitaxially deposited films can undergo the Asaro-Tiller-Grinfeld instability [1] which depends on the competition between surface and elastic energies. In addition to the influence of strain, the experimental studies show that anisotropic surface energy plays an important role as well. A dense array of pyramids has been observed, e.g. in experiments on $\mathrm{Si} / \mathrm{Ge}[2]$ and $\mathrm{SiGe} / \mathrm{Si}[3]$. In agreement with such observations, we extend the phase field model discussed in $[4,5]$ by incorporating the anisotropy in surface energy by means of the Finsler geometry as in $[6,7]$.

\section{Description of the model}

Within the context of epitaxy, consider the system consisting of solid and vapor phase. The spatial position of phases and of the transition region between them are described by the phase-field function $p=p(t, x)$ which attains the values 1 in the solid, 0 in the liquid, and values in between throughout the transition layer. In what follows, the Einstein summation is used.

The isotropic phase-field model for heteroepitaxial growth discussed in $[4,5]$ has the form

$$
\begin{aligned}
& k \rho \frac{\partial p}{\partial t}=C_{1} \nabla^{2} p-\frac{2 C_{1}}{\xi^{2}} g^{\prime}(p) \\
& -\frac{1}{\xi} h^{\prime}(p)\left(\mu\left(\epsilon_{i j}-\epsilon_{p}^{m}\right)\left(\epsilon_{i j}-\epsilon_{p}^{m}\right)+\frac{\lambda}{2}\left(\epsilon_{i i}-\epsilon_{p}^{m}\right)^{2}\right),
\end{aligned}
$$

where $k$ is the inverse mobility, $\rho$ - the solid-phase density, $C_{1}$ is the surface tension, $\xi$ is the diffuse-interface thickness, $\epsilon_{i j}=\frac{1}{2}\left(\frac{\partial u_{i}}{\partial x_{j}}+\frac{\partial u_{j}}{\partial x_{i}}\right)$ is the strain tensor with $\boldsymbol{u}$

${ }^{*}$ corresponding author; e-mail: hoangdieu@fjfi.cvut.cz as the displacement vector, $\mu$ and $\lambda$ are the Lamé constants of the solid, $\epsilon_{p}^{m}$ is the misfit, $\delta_{i j}$ is the Kronecker delta, and the functions $h^{\prime}(p)=6 p(1-p)$, and $g^{\prime}(p)=2 p(1-p)(1-2 p)$.

The stress tensor $\sigma_{i j}$ in the elastic energy density is expressed by the Hooke law with the lattice misfit

$$
\sigma_{i j}=2 \mu_{p} \epsilon_{i j}+\lambda_{p} \epsilon_{k k} \delta_{i j}+\epsilon_{p}^{m}\left(3 \lambda_{p}+2 \mu_{p}\right) \delta_{i j},
$$

where $\mu_{p}, \lambda_{p}$, and $\epsilon_{p}^{m}$ are the functions of the phase field $p$ given as follows:

$$
\mu_{p}=\mu h(p)+\mu^{v}, \quad \lambda_{p}=\lambda h(p), \quad \epsilon_{p}^{m}=\epsilon^{m} h(p),
$$

with $h(p)=p^{2}(3-2 p)$ and $\mu^{v}$ describing stress-strain relationship in vapor. The momentum conservation corresponding to the energy relaxation reads as

$$
\nabla \cdot \sigma_{i j}=0
$$

with corresponding boundary conditions given in Sect. 3 .

The anisotropic surface energy is incorporated into the model by means of the framework introduced in [6] and used in, e.g. $[7,8]$. For this purpose a continuous function $\phi: \mathbb{R}^{2} \rightarrow \mathbb{R}_{0}^{+}$called the Finsler metric is used, which satisfies the properties such as $\phi \in C^{3+\alpha}\left(\mathbb{R}^{2} \backslash\{0\}\right), \phi^{2}$ being strictly convex, $\phi(t \boldsymbol{\eta})=|t| \phi(\boldsymbol{\eta}), t \in \mathbb{R}, \boldsymbol{\eta} \in \mathbb{R}^{2}$, $\widehat{\lambda}|\boldsymbol{\eta}| \leq \phi(\boldsymbol{\eta}) \leq \widehat{\Lambda}|\boldsymbol{\eta}|, \boldsymbol{\eta} \in \mathbb{R}^{2}$, for two for suitable positive constants $0<\hat{\lambda} \leq \hat{\Lambda}$. This function plays the role of distance measurement distinguishing directions. Using function $\Phi$, we define the anisotropic operator $T: \mathbb{R}^{2} \rightarrow \mathbb{R}^{2}$ as

$$
T(\boldsymbol{\eta})=\phi(\boldsymbol{\eta}) D \phi(\boldsymbol{\eta}) \quad \text { for } \quad \eta \neq 0, \quad T(0)=0,
$$

where the $D \Phi=\left[\partial_{\eta_{1}} \Phi, \partial_{\eta_{2}} \Phi\right]$ is the total differential of $\Phi$. Operator $T$ modifies the gradient $\nabla p$ with respect to its direction when $\boldsymbol{\eta}$ being substituted by $\nabla p$.

This allows to suggest the anisotropic form of Eq. (2.1) for the thin-layer growth as

$$
\begin{aligned}
& k \rho \frac{\partial p}{\partial t}=C_{1} \nabla \cdot T(\nabla p)-\frac{2 C_{1}}{\xi^{2}} g^{\prime}(p) \\
& \quad-\frac{1}{\xi} h^{\prime}(p)\left(\mu\left(\epsilon_{i j}-\epsilon_{p}^{m}\right)\left(\epsilon_{i j}-\epsilon_{p}^{m}\right)+\frac{\lambda}{2}\left(\epsilon_{i i}-\epsilon_{p}^{m}\right)^{2}\right)
\end{aligned}
$$

endowed with the corresponding boundary and initial 
conditions given in Sect. 3 and solved in the spatial domain $\Omega=(0, L) \times(0, L)$ and in the time interval $\left(0, T_{m}\right)$.

For computational convenience, it is suitable to perform the dimensional analysis of the model Eqs. (2.3) and (2.2). For this purpose, we introduce the spatial scale $L$ and the time scale $\tau$ and substitute for the variables $x=L x^{\prime}, t=\tau t^{\prime}$ and correspondingly for $\xi=L \xi^{\prime}$. In particular, Eq. (2.3) becomes

$$
\begin{aligned}
& \frac{L^{2} k \rho}{C_{1} \tau} \frac{\partial p}{\partial t^{\prime}}=\nabla^{\prime} \cdot T\left(\nabla^{\prime} p\right)-\frac{2}{\xi^{\prime 2}} g^{\prime}(p)-\frac{1}{\xi^{\prime}} h^{\prime}(p) \\
& \quad \times\left(\frac{L}{C_{1}} \mu\left(\epsilon_{i j}-\epsilon_{p}^{m}\right)\left(\epsilon_{i j}-\epsilon_{p}^{m}\right)+\frac{L}{C_{1}} \frac{\lambda}{2}\left(\epsilon_{i i}-\epsilon_{p}^{m}\right)^{2}\right),
\end{aligned}
$$

and is solved in the spatial domain $(0,1) \times(0,1)$ and in the time interval $\left(0, T_{m} / \tau\right)$. Computational results are then re-scaled back to real variables and presented in Sect. 3 .

\section{Computational results}

The system of model equations is solved in the square domain $\Omega=(0, L) \times(0, L)$ and within the time interval $\left(0, T_{m}\right)$, where $L=1 \times 10^{-6} \mathrm{~m}$ and $T_{m}=600 \mathrm{~s}$, or $300 \mathrm{~s}$, respectively. The bottom part of $\Omega$ is filled by solid $(p=1)$ whereas the upper part with vapor $(p=0)$. On the bottom edge of $\Omega$ the boundary conditions are prescribed as $p=1$ and $\boldsymbol{u}=0$. On the upper edge of $\Omega$ the boundary conditions are prescribed as $p=0$ and $\sigma \cdot \boldsymbol{n}=0$. Periodic boundary conditions are applied at the lateral boundaries. The parameters are set for the $\mathrm{SiGe} / \mathrm{Si}$ system as in [3] and are summarized in the Table.

\section{TABLE}

Parameters of the $\mathrm{SiGe} / \mathrm{Si}$ system.

\begin{tabular}{c|c}
\hline \hline Parameters & Real values \\
\hline$k$ & $1.00 \times 10^{14}\left[\mathrm{~m} \mathrm{~s}^{-1}\right]$ \\
$\rho$ & $4.00 \times 10^{3}\left[\mathrm{~kg} \mathrm{~m}^{-3}\right]$ \\
$C_{1}$ & $1.00\left[\mathrm{~N} \mathrm{~m}^{-1}\right]$ \\
$\mu$ & $1.59 \times 10^{11}\left[\mathrm{~N} \mathrm{~m}^{-2}\right]$ \\
$\lambda$ & $1.48 \times 10^{11}\left[\mathrm{~N} \mathrm{~m}^{-2}\right]$ \\
$\epsilon^{m}$ & 0.01 \\
$\mu^{v}$ & $1.00 \times 10^{6}\left[\mathrm{~N} \mathrm{~m}^{-2}\right]$ \\
$\xi$ & $0.02 \times 10^{-6}[\mathrm{~m}]$
\end{tabular}

We assume that the surface energy has the cubic anisotropy characterized by the following function:

$$
\phi^{1}(\boldsymbol{\eta})=\sum_{i=1}^{2} \sqrt{\eta_{i}{ }^{2}+0.02 \sum_{j=1}^{2} \eta_{j}{ }^{2}},
$$

in correspondence to a suitable two-dimensional reproduction of the anisotropy of the above mentioned material. This anisotropy is rotated clockwise to the angle $\pi / 4$ to obtain the second example of anisotropy

$$
\phi^{2}(\boldsymbol{\eta})=\phi^{1}\left(R_{\pi / 4}(\boldsymbol{\eta})\right),
$$

with $R_{\pi / 4}$ denoting the mentioned rotation. The system of Eqs. (2.3) and (2.2) together with boundary and initial conditions is transformed into the dimensionless form as described in Sect. 2 - we set $L=1 \times 10^{-6} \mathrm{~m}$, and $\tau=4 \times 10^{5} \mathrm{~s}$. Then it is numerically solved by the explicit numerical scheme based on the finite element method. The algorithm has been implemented in FreeFem ++see [9]. The presented results were obtained on a triangular structured mesh of 10000 elements. The solution of two examples at different times for the two anisotropy examples are presented. The thin layer solid-phase boundary is identified by the level set $p(t, x)=\frac{1}{2}$ and the solid phase is located below this level set.

In Fig. 1 the anisotropy $\phi^{1}$ is used. We observed the development of facets and the surface evolves towards a rectangle-like morphology.
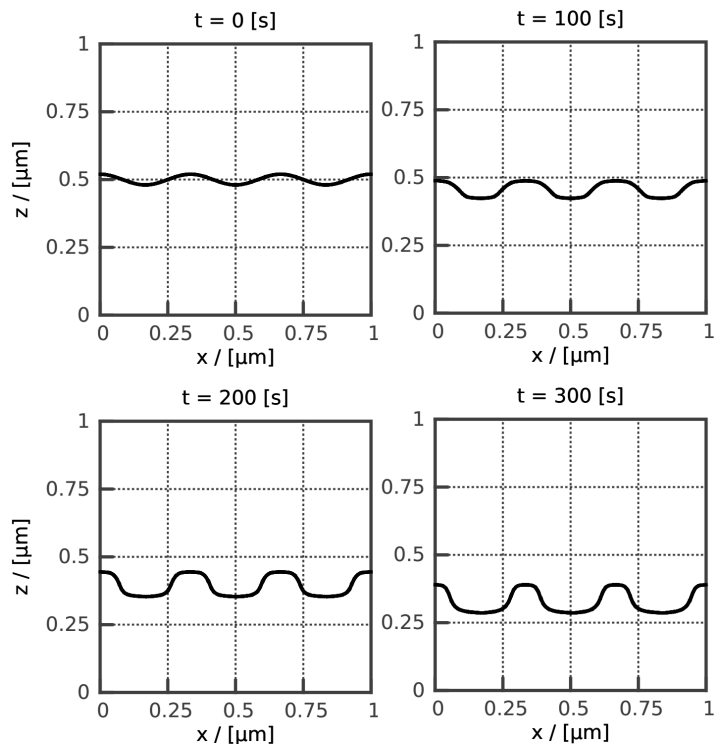

Fig. 1. Time evolution of the epitaxial layer with the anisotropy $\phi^{1}$.
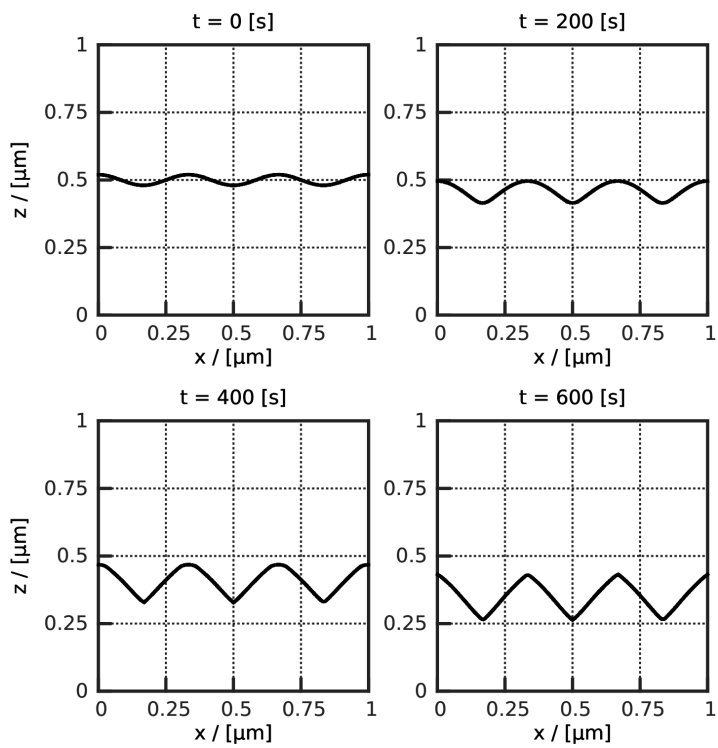

Fig. 2. Time evolution of the epitaxial layer with the anisotropy $\phi^{2}$.

For the anisotropy $\phi^{2}$ the obtained evolution is shown in Fig. 2. Faceting occurs again and the surface evolves towards a pyramidal morphology in agreement with $[2,3]$. 


\section{Conclusion}

We have presented the anisotropic phase field model for heteroepitaxial growth of $\mathrm{SiGe}$ on $\mathrm{Si}$. The numerical simulations showed the influence of surface and elastic energies which results in faceted mounds. Depending on the anisotropy type, the film evolves towards an array of rectangle-like islands or pyramids. The model can be used for other thin-film systems by defining the corresponding anisotropy and other material parameters properly.

\section{Acknowledgments}

The research has been supported by the grant "Multidisciplinary research center for advanced materials", the project No. 14-36566G of the Grant Agency of the Czech Republic.

\section{References}

[1] D.J. Srolovitz, Acta Metall. 37, 621 (1989).

[2] I. Berbezier, A. Ronda, A. Portavoce, J. Phys. Condens. Matter 14, 8283 (2002).

[3] Y.W. Zhang, A.F. Bower, J. Mech. Phys. Solids 47, 2273 (1999).

[4] H. Emmerich, Cont. Mech. Thermodyn. 15, 197 (2003).

[5] K. Kassner, C. Misbah, J. Müller, J. Kappey, P. Kohlert, Phys. Rev. B 63, 036117 (2001).

[6] G. Bellettini, M. Paolini, Hokkaido Math. J. 25, 537 (1996).

[7] M. Beneš, Appl. Math. 48, 437 (2003).

[8] D.H. Hoang, M. Beneš, T. Oberhuber, Acta Polytechn. Hung. 10, 99 (2013).

[9] F. Hecht, J. Numer. Math. 20, 251 (2012). 